\title{
Chronic chemotherapy with paclitaxel nanoparticles induced apoptosis in lung cancer in vitro and in vivo
}

This article was published in the following Dove Medical Press journal: International Journal of Nanomedicine

\author{
Xuefeng Zhao ${ }^{1,2}$ \\ Juan Fan' \\ Peng Wu' \\ Chengming $\mathrm{Wei}^{2}$ \\ Qiongying Chen ${ }^{3}$ \\ Zhi Ming ${ }^{2}$ \\ Jun Yan ${ }^{2}$ \\ Linglin Yang' \\ 'Department of Oncology, \\ The Affiliated Hospital of Southwest \\ Medical University, Luzhou 646000 , \\ China; ${ }^{2}$ Department of Oncology, \\ Zigong Ist People's Hospital, \\ Zigong 643000, China; ${ }^{3}$ Department \\ of Oncology, Zigong 3rd People's \\ Hospital, Zigong 643000, China
}

Aim: Paclitaxel (PTX) is an effective antitumor drug. Previous research demonstrated that paclitaxel nanoparticles (PTX-NPs) exhibited the greatest antitumor effect at 15 hours after light onset (15 HALO), but the mechanism in chronic chemotherapy is still unknown. In our study, we investigated whether PTX-NPs regulated Period2 (Per2) during chronic chemotherapy to induce apoptosis in vivo and in vitro.

Methods: To improve the antitumor effect and reduce organ damage induced by PTX treatment, PTX-NPs were prepared using a film dispersion method. Then, A549 cells were treated with PTX-NPs at 0, 5, 10, 15, and 20 HALO. An annexin/PI V-FITC apoptosis kit was measured for apoptosis, and PI was analyzed for cell cycle. The relative mechanism was detected by RT-PCR and Western blotting. Tumor volume and weight were measured to evaluate the antitumor effect of the PTX-NPs, and H\&E staining was performed to assess organ damage.

Results: Cell cycle analysis demonstrated that PTX-NPs blocked cell cycle in G2 phase and that the ratio of cell death was significantly increased in A549 cells, while the ratios of cells in G2 phase and of apoptotic cells were highest at 15 HALO. Evaluation of in vivo antitumor activity revealed that PTX-NPs inhibited tumor growth and decreased tumor weight at 15 HALO. RT-PCR and Western blotting demonstrated that PTX-NPs upregulated Per2 mRNA and protein expression, and the highest Per2 expression was observed at 15 HALO in vivo and in vitro. Meanwhile, Bax mRNA and protein expression was upregulated, while Bcl-2 mRNA and protein expression was downregulated after PTX-NPs treatment in vivo. Moreover, H\&E staining revealed that PTX-NPs reduced liver damage at 15 HALO.

Conclusion: PTX-NPs exhibited the most effective antitumor activity and reduced liver damage at 15 HALO through upregulation of Per2 expression to induce apoptosis in vivo and in vitro. Keywords: paclitaxel nanoparticles, chronic chemotherapy, Per2, apoptosis, lung cancer

\section{Introduction}

Over the past century, lung cancer has gone from being the rarest of diseases to one of the greatest causes of cancer-related deaths in men worldwide and, in some parts of the world, in women. ${ }^{1-5}$ In 2012, nearly 1.6 million people died from lung cancer. Lung cancer is frequently caused by tobacco smoking, which has been confirmed in many studies, both ecological and clinical. ${ }^{4}$ Lung cancer treatment strategies are typically surgery, radiotherapy, and chemotherapy, which are effective but have serious side effects. Thus, it is of great significance to explore more efficient therapeutic strategies for the treatment of lung cancer.

Paclitaxel (PTX) has been widely used in the clinic against human cancer. In clinical trials, PTX appears to be active against a number of human malignancies, including 
non-small-cell lung cancer and breast cancer. ${ }^{6,7}$ Moreover, in a preclinical study in mouse xenograft tumor models, PTX was active against various human tumor cell lines, including melanoma and colon adenocarcinoma cells. ${ }^{8}$ Although PTX exhibits effective antitumor activity, the major issues we are facing in cancer therapy with PTX are drug resistance and severe side effects. ${ }^{9}$ Therefore, finding a new treatment method is necessary to solve these issues.

Drug solubility is a necessary point in drug development. A water-insoluble drug makes their formulation difficult, which greatly limited their further clinical use. The nanoparticulate system for drug delivery will be promising to solve the above-mentioned issue. Encapsulation of active components into polymeric carriers become a hopeful method of preventing drug degradation, increasing drug bioavailability, decreasing drug toxic effects, achieving specific targeting, and controlling drug release. Recently, many new PTX-nanoparticles have appeared around the world. Polymeric nanoparticles have numerous benefits in drug delivery systems: ${ }^{10}$ first of all, it has stronger antidilution and it is stable in the circulating system, second, its cover nanosize and hydrophilic nature might prevent reticuloendothelial system recognition during absorption in the body, thus extending the time in the blood circulation system. Finally, due to the enhancement permeability and retention, a small size of nanoparticles $(10-200 \mathrm{~nm})$ is beneficial to the tumor tissue accumulation and retention, considering their size to be ideal for passive targeting of tumor tissue. As a result, polymeric nanoparticles exhibit a promising drug delivery system for anti-cancer drugs. ${ }^{11}$ Poly(e-caprolactone)poly(ethyleneglycol)-poly(e-caprolactone) is a synthesized tri-block copolymer, which belongs to the class of hydrolytically degradable polymers. Because of its appropriate mechanical properties, amphiphilic properties, biodegradability, and biocompatibility, it is widely used as carrier of a number of antitumor drugs. ${ }^{12,13}$

Timely chemotherapy dependent on biological rhythms is used to treat cancer in the clinic. ${ }^{14}$ Several chemotherapy drugs have been used to kill cancer, such as 5-FU, cyclophosphamide, and PTX. ${ }^{15}$ However, not only does timely chemotherapy improve the treatment effect, but it also might reduce toxicity and side effects, including myelosuppression. ${ }^{16}$ Therefore, timely chemotherapy is the preferred treatment method.

The circadian timing system not only regulates cell death and DNA repair, but also controls certain signaling and metabolic pathways that are relevant in cancer processes and treatments. ${ }^{17,18}$ Disorder of the circadian rhythm can disrupt normal biological processes and result in pathologic conditions, such as cancer, metabolic syndrome, cardiovascular disease, and manic depression. ${ }^{19}$ Light plays a crucial role in entraining circadian timing. Light was thought to synchronize the molecular oscillations by acting to change the levels of the clock component. In mammals, light acts to rapidly induce level of clock gene transcripts and regulates related "clock molecules" expression, ${ }^{20}$ such as Per2. Per2, a core gene component of the circadian clock, is closely related to the development and occurrence of tumors; common malignant tumors including neuroglioma, pancreatic cancer, and non-small-cell lung cancer exhibit a deficiency and reduction in Per2 expression. ${ }^{21}$ In addition, Per2 has tumor suppressor properties and is mutated or downregulated in human breast cancer. ${ }^{22}$ Therefore, Per2 is a potential target for cancer treatment.

Chronic chemotherapy has become an effective antitumor method. In our previous study, we demonstrated that paclitaxel nanoparticles (PTX-NPs) exhibited greater antitumor activity against A549 cells in vivo and exerted the strongest antitumor effect at 15 hours after light onset (HALO). ${ }^{23}$ Moreover, a previous study demonstrated that cell viability at $15 \mathrm{HALO}$ was the lowest among the four time points, suggesting that the cytotoxicity of PTX-NPs against A549 cells was related to circadian variation. However, the mechanism by which PTX-NPs exert anticancer activity in chronic chemotherapy remains unknown. To investigate the role of Per2 in PTX-NPs cancer treatment, the present study used A549 cells as the working system. First, cell cycle and apoptosis of A549 cells after PTX-NPs treatment were detected via flow cytometry methods. Subsequently, the mRNA expression of Bax, Bcl-2, and Per2 in A549 cells was determined using RT-PCR. The anticancer effect of the PTX-NPs on A549 tumors transplanted into nude mice was investigated. In addition, Western blot analyses were performed to identify the roles of Per2, Bax, and Bcl-2 in apoptosis after PTX-NPs treatment.

\section{Materials and methods Materials}

PTX (98\%) was purchased from Chengdu Manchester Biotechnology Co., Ltd. (Chengdu, China). $\varepsilon$-Caprolactone ( $\varepsilon-C L)$ was obtained from Alfa Aesar (Ward Hill, MA, USA). MPEG $(\mathrm{Mn}=2,000)$, stannous octoate $\left[\mathrm{Sn}(\mathrm{Oct})_{2}\right]$, dimethyl sulfoxide (DMSO), methyl alcohol, and acetonitrile (HPLC grade) were obtained from Kelong Co., Ltd. (Chengdu, China).

\section{Cell lines and animals}

Human lung carcinoma A549 cell line used in this study were purchased by the Experimental Research Center of 
the Affiliated Hospital of Southwest Medical University (Luzhou, China) from the Cell Bank of the Chinese Academy of Sciences, and cultured in Roswell Park Memorial Institute (RPMI) medium 1,640 (Gibco, Waltham, MA, USA) supplemented with $10 \%$ fetal bovine serum (FBS, Gibco), $100 \mu \mathrm{g} / \mathrm{mL}$ streptomycin sulfate, and $100 \mathrm{IU} / \mathrm{mL}$ penicillin at $37^{\circ} \mathrm{C}$ under a $5 \% \mathrm{CO}_{2}$ atmosphere and high humidity.

$\mathrm{Balb} / \mathrm{c}$ null mice (female, 4 weeks old, 16-20 g) were supplied by Chengdu Dossy Experimental Animals Co., Ltd. (Chengdu, China, license no SCXK 2013-24) and housed at a controlled temperature of $20^{\circ} \mathrm{C}-26^{\circ} \mathrm{C}$, with a relative humidity of $40 \%-70 \%$ and 12 -hour light/dark cycles. The mice were provided a standard diet of laboratory chow and tap water ad libitum. Animal use was in accordance with Ethical and Welfare in Animal Experimentation of Southwest Medical University, and the protocol was approved by the Institutional Animal Care and Treatment Committee of Southwest Medical University (Luzhou, China).

\section{Synthesis of PCEC tri-block polymer}

PCEC tri-block polymer was synthesized using the ringopening polymerization method. Briefly, a predetermined amount of $\varepsilon-C L$ and PEG was placed successively into a dry flask under the protection of flowing nitrogen and with the addition of a few drops of $\mathrm{Sn}(\mathrm{Oct})_{2}$. Then, the flask was gently stirred in a $130^{\circ} \mathrm{C}$ oil bath. The synthetic polymer was cooled to room temperature in a nitrogen atmosphere for 6 hours. PCEC polymer was then dissolved in dichloromethane and precipitated with AR-grade excess cold petroleum ether. In the end, the purified mixture was dried in a $37^{\circ} \mathrm{C}$ oven to a constant weight under vacuum conditions and placed into a sealed bag for subsequent use.

\section{Preparation of PTX-NPs}

PTX-NPs were prepared using the film dispersion method. First, $12 \mathrm{mg}$ PCEC and $108 \mathrm{mg}$ PCEC copolymers were dissolved in acetone solution, and the solvent was removed using a rotary evaporator to prepare PTX/PCEC at $37^{\circ} \mathrm{C}-40^{\circ} \mathrm{C}$. The PTX/PCEC was dissolved in ultrapure water. Then, the composite solution was filtered (220 nm, Millipore, Sigma, Burlington, MA, USA) to remove the nonencapsulated PTX. Finally, the filtrate was freeze-dried to obtain PTX-NP powder.

\section{Detection of PTX content in the nanoparticles}

The drug loading (DL) efficiency of the PTX-NPs was determined using high-performance liquid chromatography (HPLC, Agilent, 1260, Agilent Technologies, Santa Clara, CA, USA). The mobile phase was a mixture of acetonitrile, methanol, and water $(2: 1: 2)$, with a flow rate of $1 \mathrm{~mL} / \mathrm{min}$. The reverse-phase column was a $\mathrm{C} 18$ column $(4.6 \times 150 \mathrm{~mm}$, $3.5 \mu \mathrm{m}$ particle size). The column temperature was maintained at $35^{\circ} \mathrm{C}$, and the detection wavelength was set to $227 \mathrm{~nm}$. The standard curve for drug detection was established and was used to calculate the drug loading efficiency of PTX according to the following formula:

$$
\text { DL }(\%)=\frac{\text { Amount of PTX }}{\text { Amount of polymer }+ \text { PTX }} \times 100 \%
$$

\section{Determination of PTX-NPs size and morphology}

The particle size (diameter, nm) and surface charge (zeta potential, $\mathrm{mV}$ ) of the PTX-NPs were assessed by dynamic light scanning (DLS; NanoBrook90Plus Zeta, Brookhaven, NY, USA). The morphology was viewed via transmission electron microscopy (TEM; Tecnai G2F20, FEI, Hillsboro, OR, USA). The PTX-NPs were stained with phosphotungstate stain before TEM imaging.

\section{Cell cycle analysis}

Cell cycle analysis was performed as described previously. ${ }^{24}$ The cells were seeded in 6 -well plates $\left(1 \times 10^{5}\right.$ per well) and grown overnight at $37^{\circ} \mathrm{C}$ in a humidified incubator with $5 \% \mathrm{CO}_{2}$. Cells were then treated with PTX and PTX-NPs at the indicated concentration at $0,5,10,15$, and 20 HALO. The cells were then washed and harvested (including attached and detached cells) and fixed with $75 \%$ alcohol at $4{ }^{\circ} \mathrm{C}$. After fixation, the cells were washed with phosphate-buffered saline (PBS) and stained with PBS containing $50 \mathrm{mg} / \mathrm{mL}$ PI and $100 \mathrm{mg} / \mathrm{mL}$ RNase A for 30 minutes. The cell cycle distribution was determined via flow cytometry analysis with the aforementioned FACSCalibur device and analyzed using CellQuest software.

\section{Cell apoptosis analysis}

The cells were treated with $27.6 \mu \mathrm{g} / \mathrm{mL}$ (containing $2.5 \mu \mathrm{g} / \mathrm{mL}$ PTX) PTX-NPs at 0, 5, 10, 15, and 20 HALO. Then, they were harvested and washed with PBS. Apoptotic cells were determined with a FITC Annexin V Apoptosis Detection Kit (BD, Franklin Lakes, NJ, USA) according to the manufacturer's protocol. Apoptosis was analyzed with a fluorescence microscope and FACScan laser flow cytometer. The data were analyzed using CELLQuest software.

\section{RT-PCR analysis}

Total RNA from A549 cells was isolated according to the Total RNA Extraction Reagent (Takara, Beijing, China) 
protocol. Analysis was carried out with a StepOne ${ }^{\mathrm{TM}}$ PCR System. The sequences of primers were as follows: Forward 5'-CTATTCTCCCATTCGGTTTCG-3' and Reverse 5'-CACCCTGACTTTGTGCCTCC-3' for Per2, Forward 5'-TGTCGCCCTTTTCTACTTTGC-3' and Reverse 5'-GAGGCTTGAGGAGTCTCACCC-3' for Bax, Forward 5'-ACATCGCCCTGTGGATGACT-3' and Reverse 5'-AGGGCCAAACTGAGCAGAGTC-3' for Bcl-2, and Forward 5'-CATCATCCCTGCCTCTACTGG-3' and Reverse 5'-GTGGGTGTCGCTGTTGAAGTC-3' for GAPDH (as the reference gene). Per2, Bax, and Bcl-2 mRNA expression levels were analyzed using the $2^{-(\triangle \Delta \mathrm{CT})}$ method.

\section{Western blotting}

A549 cells $\left(1 \times 10^{5}\right.$ cells per well) were seeded into 6-well plates. After 12 hours of incubation, the seeded cells were treated with PTX or PTX-NPs at 0, 5, 10, 15, and 20 HALO and incubated at $37^{\circ} \mathrm{C}$ in a $5 \% \mathrm{CO}_{2}$ atmosphere. Total protein was lysed using $1 \times$ radioimmunoprecipitation (RIPA) lysis buffer on ice and then centrifuged at $12,000 \mathrm{rpm}$ at $4^{\circ} \mathrm{C}$ for 20 minutes. The sample supernatants were collected to detect protein concentration using a Bradford assay. Loading buffer was added to the sample supernatants, and the proteins were denatured at $96^{\circ} \mathrm{C}$ for 5 minutes. The proteins were separated via sodium dodecyl sulfate-polyacrylamide gel electrophoresis (SDS-PAGE) and electrically transferred onto a PVDF membrane using $1 \times$ transfer buffer (Invitrogen, Carlsbad, CA, USA). Afterwards, the membrane was blocked in PBST containing 5\% nonfat milk for 1 hour and incubated with primary antibody at $4^{\circ} \mathrm{C}$ overnight, followed by incubation with HRPconjugated secondary antibody for 40 minutes at room temperature. The incubation with primary antibody was performed as follows: anti-Per2 antibody diluted to 1:1,000 was incubated with A549 cell immunoblots, and the results were developed with the ECL reagent (Aspen, Durban, South Africa). The protein band intensity was quantified using ImageJ.

\section{In vivo animal tumor model experiment}

To assess the effect of chronic chemotherapy with PTX-NPs, antitumor activity was evaluated against A549-transplanted null mice. A549 xenograft mouse models were established through hypodermic injection of A549 cells $\left(1 \times 10^{7}\right.$ cells $/ \mathrm{mL}$ in $100 \mu \mathrm{L}$ of sterile PBS) in the dorsal aspect of the right thigh of mice. Tumors were established for approximately 14 days to reach an approximate tumor volume of $100 \mathrm{~mm}^{3}$ before treatment. The mice were randomly divided into three groups: saline group, PTX group, and PTX-NP group. Then, each group was further divided into five groups: 0 HALO,
5 HALO, 10 HALO, 15 HALO, and 20 HALO. The saline group was used as a control group, and the other groups were considered treatment groups. The PTX and PTX-NPs solution were injected through the tail vein every 3 days (three times) at a PTX dose of $10 \mathrm{mg} / \mathrm{kg} /$ day for the PTX and PTX-NPs groups; the control group was given an equal volume of saline. The tumor volumes were measured every other day with Vernier calipers. Tumor volume (V) was calculated using the formula $\mathrm{V}=\left(\mathrm{ab}^{2}\right) / 2$, where a is the major length axis and $b$ is the minor length axis. After 12 days of treatment, the mice were sacrificed, and the tumor tissue was completely removed. RT-PCR and Western blotting were used to detect the expression of mRNA and protein in vivo. Then, the tumor growth inhibition rate (IR) was calculated using the following formula:

$\mathrm{IR}=$

1 - the average tumor weight of the treatment group

The average tumor weight of the control group

\section{Hematoxylin/eosin (H\&E) staining}

Tumor transplanted nude mice were euthanized on the 12th day, and the heart, liver, spleen, lung, and kidney were collected. The tissues were fixed with $4 \%$ formaldehyde, embedded in paraffin, and cut into sections $(5 \mathrm{~mm})$ for $\mathrm{H} \& \mathrm{E}$ staining.

\section{Statistical analysis}

All parameter values are reported as the mean \pm standard deviation (mean $\pm \mathrm{SD}$ ). The statistical significance of the data was determined via one-way univariate analysis of variance (ANOVA) for comparing the means of more than two independent groups. A difference with $P<0.05$ was considered to be statistically significant (marked as *). A higher significance level was set at $P<0.01$ (marked as **).

\section{Results}

\section{Determination of PTX-NPs size and morphology}

PTX-NPs were produced by the film dispersion method. As shown in Figure 1, TEM measurement of the phosphotungstate-stained PTX-NPs showed that they were composed of a hydrophobic core and a hydrophilic shell. Our results showed that PTX-NPs had a size of $174.9 \pm 6.5 \mathrm{~nm}$. Moreover, the theoretical drug loading of the nanoparticles was recorded as $10 \%$. According to HPLC data, the DL content of PTX-NPs was $9.60 \% \pm 0.61 \%$. 


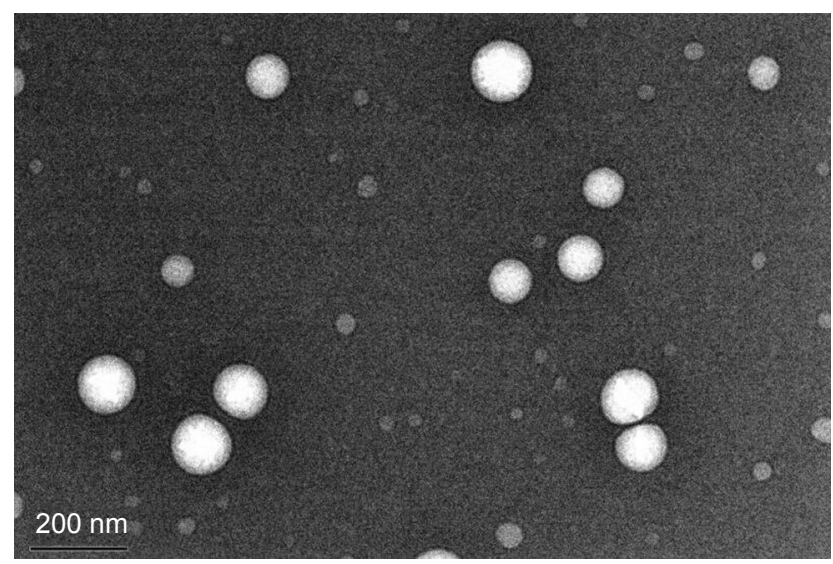

Figure I TEM image of PTX-NPs (magnification $\times 12,000$ ).

Abbreviations: PTX-NPs, paclitaxel nanoparticles; TEM, transmission electron microscopy.

\section{PTX-NPs inhibited cell cycle arrest and induced apoptosis}

To investigate the effect of chronic chemotherapy with PTXNPs on the A549 cell cycle, cell cycle distribution and apoptosis were determined via flow cytometry. Figure 2 shows that the PTX-NPs and PTX treatment inhibited cell cycle arrest and induced apoptosis. PTX-NPs might block the cell cycle in $\mathrm{G} 2$ phase at different treatment times. However, the highest percentage of A549 cells in G2 phase was $82.32 \% \pm 5.97 \%$ at 15 HALO. Moreover, the apoptosis analysis revealed that the PTX-NPs might induce cell apoptosis in the A549 cells; the percentages of A549 cells that died were $14.62 \% \pm 0.83 \%$, $20.15 \% \pm 2.30 \%, 30.92 \% \pm 2.13 \%, 50.16 \% \pm 3.72 \%$, and $29.29 \% \pm 2.53 \%$ at $0,5,10,15$, and $20 \mathrm{HALO}$, respectively.

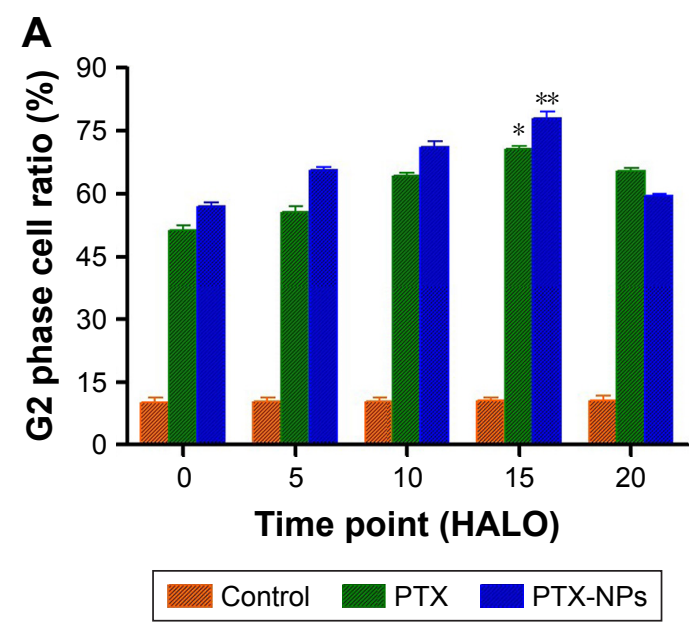

Our results showed that the A549 cell apoptosis ratio was highest after PTX-NPs treatment at 15 HALO.

\section{PTX-NPs upregulated Per2 gene and protein expression in vitro}

To further understand the mechanism by which PTX-NPs induced A549 cell apoptosis, Per2 gene and protein expression was detected via RT-PCR and Western blotting. As shown in Figure 3, the expression of Per2 increased after PTX-NPs treatment before 20 HALO. However, the expression of Per2 was downregulated after PTX-NPs treatment at 20 HALO, and the Per 2 expression was highest at 15 HALO. Our results demonstrated that PTX-NPs treatment of A549 cells at 15 HALO had a stronger effect on upregulation of Per2 gene and protein expression.

\section{In vivo study of PTX-NPs inhibition of lung cancer xenograft development}

The antitumor effect of PTX-NPs in chronic chemotherapy was further evaluated with transplanted A549 cell xenografts in nude mice. The tumor volumes in various groups were measured and are reported in Figure 4. Compared to the Control group, the PTX and PTX-NPs groups had remarkably reduced tumor volume. To further compare the effect of treatment timing on antitumor activity, PTX and PTX-NPs were administered to mice at different times. The results revealed that administration at $15 \mathrm{HALO}$ induced the best tumor growth inhibition rate by PTX-NPs. At the end of the experiment, the tumors were removed from the mice and measured, and the tumor inhibition rates of PTX-NPs

\section{B}

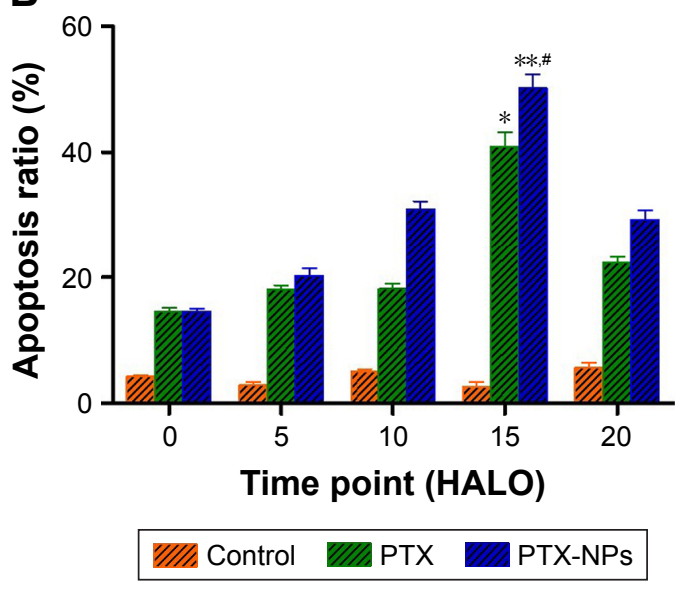

Figure 2 Effect of PTX-NPs on cell cycle and apoptosis of A549 cells, as determined by flow cytometry analysis.

Notes: (A) Representative histograms depicting cell cycle distribution in A549 cells treated with PTX and PTX-NPs at the indicated concentrations at 0,5 , 10 , I5, and 20 HALO; (B) The percentages of cell death in A549 cells treated with PTX and PTX-NPs at the indicated concentrations at 0, 5, I0, I5 and 20 HALO. Compared to control, $* P<0.05$, and $* * P<0.01$; compared to PTX, ${ }^{*} P<0.05$.

Abbreviations: HALO, hours after light onset; PTX, paclitaxel; PTX-NPs, paclitaxel nanoparticles; TEM, transmission electron microscopy. 

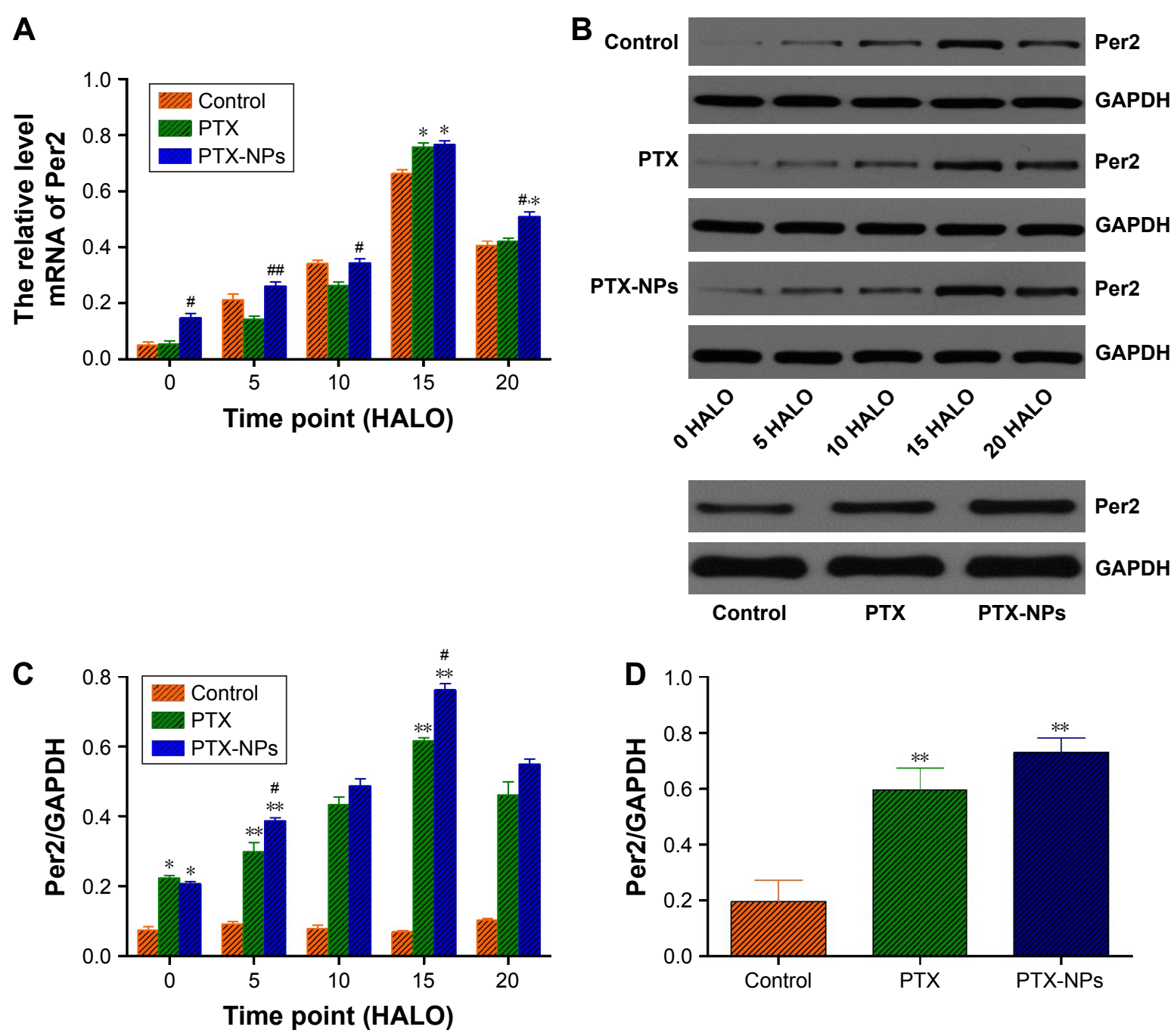

Figure 3 Per2 mRNA and protein expression after PTX-NPs treatment at 0, 5, 10, 15, and 20 HALO.

Notes: (A) The relative Per2 mRNA expression after PTX-NPs treatment at 0, 5, 10, 15, and 20 HALO; (B) Per2 expression was determined by Western blotting analysis; (C) The protein expression level of Per2 was analyzed by Imagej software; (D) the protein expression level of Per2 was analyzed by Image software. Compared to control, $* P<0.05$, and $* * P<0.01$; compared to $P T X,{ }^{*} P<0.05$; $\# P<0.01$.

Abbreviations: HALO, hours after light onset; PTX, paclitaxel; PTX-NPs, paclitaxel nanoparticles.

administered at $0,5,10,15$, and 20 HALO were $56.63 \%$, $57.63 \%, 59.93 \%, 68.54 \%$, and $58.43 \%$, respectively. The results demonstrated that the tumor inhibition rate of PTX and PTX-NPs followed a decreasing magnitude: 15 HALO $>20$ HALO $>10$ HALO $>5$ HALO $>0$ HALO. In conclusion, our study showed that PTX and PTX-NPs had the best inhibition effect at 15 HALO.

\section{Chronic chemotherapy regulated Per2, $\mathrm{Bax}$, and $\mathrm{Bcl}-2$ gene, and protein expression in vivo}

To reveal the effect of chronic chemotherapy on apoptosis, Per2, Bax, and Bcl-2 mRNA, and protein expression were detected by RT-PCR and Western blotting in vivo. As described in Figure 5, the mRNA expression of Bax was upregulated following time-point treatment, and Bax mRNA expression was highest at 15 HALO and decreased at 20 HALO. However, the mRNA expression of Bcl-2 exhibited a trend opposite to that of Bax mRNA. Our experiments showed that PTX-NPs induced the most apoptosis at 15 HALO. Moreover, Figure 6 shows that the protein expression of Per2, Bax, and Bcl-2 was consistent with the mRNA expression.

\section{Chronic chemotherapy with PTX-NPs reduced the number of liver lesions}

Enhancement of the chemotherapeutic effect of PTX-NPs may increase the risk of side effects. Therefore, we investigated the side effects of PTX-NPs on the major organs. In our study, the heart, liver, spleen, lung, and kidney were 
A

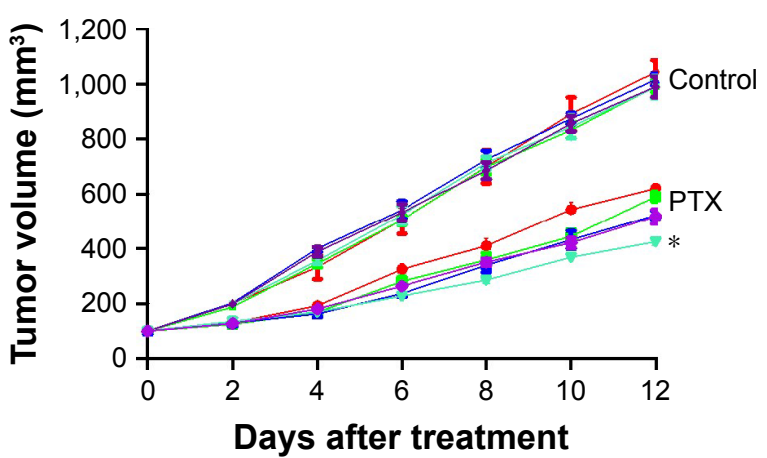

B

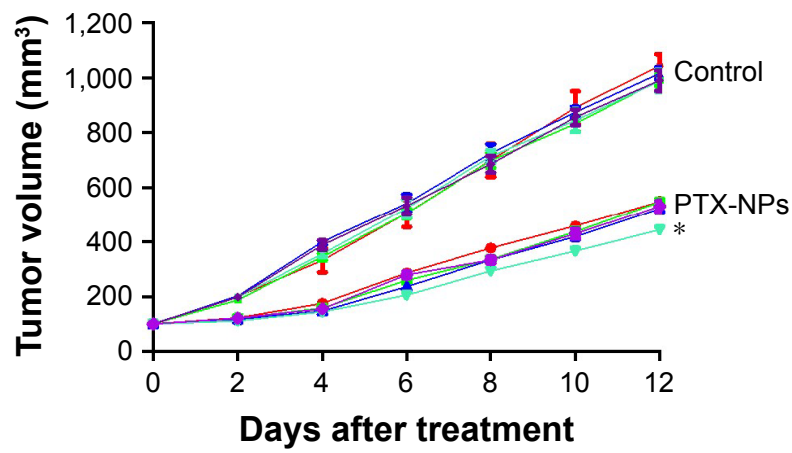

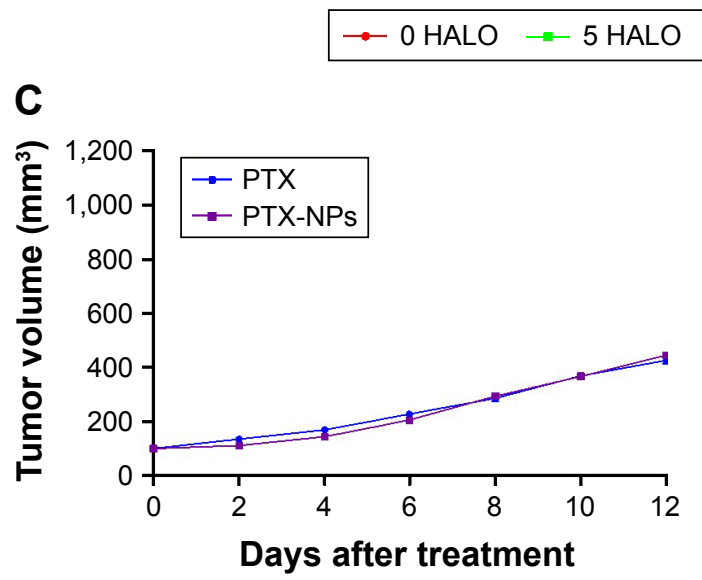

Figure 4 Antitumor effect of PTX-NPs on A549-transplanted null mice.

Notes: (A) Compared to tumor volume between control and PTX groups at different time points; (B) Compared to tumor volume between control and PTX-NPs groups at different time points; (C) Compared to tumor volume between PTX and PTX-NPs groups at I5 HALO; (D) Tumor growth inhibition rate (\%) after injection with PTX or PTX-NPs. $* P<0.05$. Mice treated with saline were used as controls.

Abbreviations: HALO, hours after light onset; PTX, paclitaxel; PTX-NPs, paclitaxel nanoparticles.

examined via $H \& E$ staining. Our results revealed that mice treated with PTX-NPs had no lesion in the heart, spleen, lung, or kidney. As shown in Figure 7, the PTX-NPs group exhibited liver damage. However, liver damage was relatively small in the 15 HALO group. Therefore, it is critical to choose the right administration time to reduce liver damage.

\section{Discussion}

One third of all cancer-related deaths are due to lung cancer, which accounts for more deaths each year than breast, prostate, and colon cancer combined. ${ }^{25-27}$ The median survival of patients with untreated metastatic non-small-cell lung cancer (NSCLC) is only 4-5 months, with a survival rate at 1 year of only $10 \% .{ }^{28}$ Chemotherapy for advanced NSCLC is often considered ineffective or excessively toxic. Several chemotherapy drugs have been used to treat cancer in the clinic, such as paclitaxel and 5-FU. Paclitaxel is effective for treating NSCLC ${ }^{29}$ However, some NSCLC tumors are not sensitive to paclitaxel treatment, and the treatment has serious side effects. ${ }^{30}$ Therefore, choosing the optimal dosage and timing of administration to reduce side effects is a promising approach. Chronic chemotherapy is a new and effective treatment method. ${ }^{31}$ Meanwhile, the concrete mechanism of action of PTX-NPs on A549 cells has never been explored in detail in chronic chemotherapy.

Although PTX-NPs significantly blocked cell cycle and inhibited the growth of tumors derived from A549 cell lines in A549-transplanted nude mice, they exhibited the best anticancer effect at 15 HALO. Based on these phenomena, the regulatory effects of PTX-NPs on specific signaling pathways of anticancer activity were investigated. Apoptosis is programmed cell death that involves the mitochondrial apoptosis pathway. PTX has been reported to potentially induce cell death through the mitochondrial apoptosis pathway. ${ }^{32,33}$ However, our data demonstrated that PTX-NPs might also block cell cycle progression, activate Bax, and inhibit Bcl-2 to induce apoptosis in vivo and in vitro, which is consistent with previous studies. ${ }^{34,35}$ Meanwhile, our study revealed 

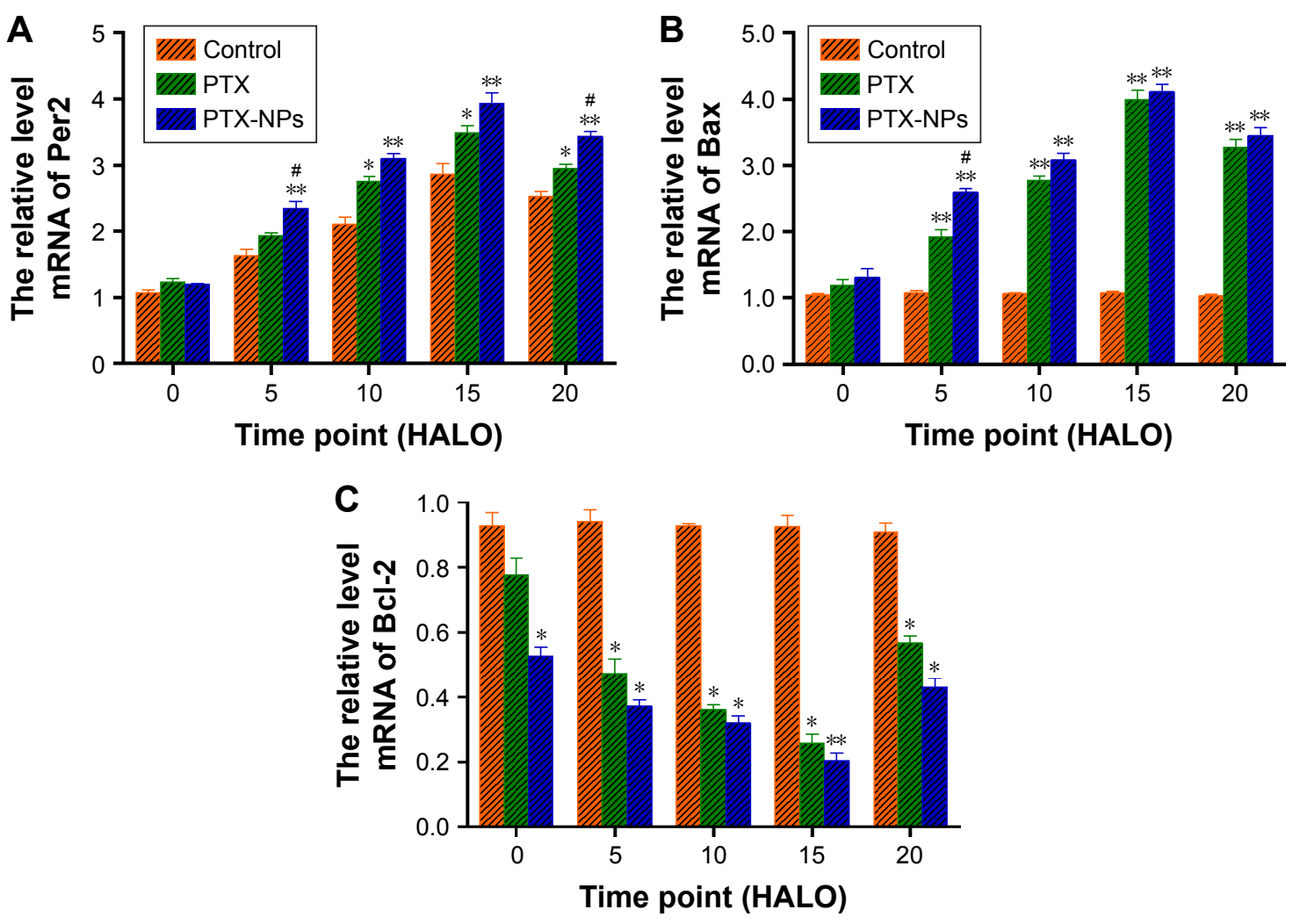

VIIA Control VIA PTX PTX-NPs

Figure 5 The relative expression of Per2, Bax, and Bcl-2 mRNA in A549-transplanted null mice after PTX-NPs treatment at 0, 5, 10,15 , and 20 HALO in vivo. Notes: (A) The relative mRNA level of Per2; (B) The relative mRNA level of Bax; (C) The relative mRNA level of Bcl-2. Compared to control, $* P<0.05$, and $* * P<0.01$; compared to PTX, ${ }^{P}<0.05$. Mice treated with saline were used as controls.

Abbreviations: HALO, hours after light onset; PTX, paclitaxel; PTX-NPs, paclitaxel nanoparticles.

that PTX and PTX-NPs have the best anticancer effect at 15 HALO, and the mRNA and protein expression of Per2 was the highest at this time point.

Per is a core clock gene that regulates the proliferation, secretion, and metabolism of normal cells and the circadian rhythm of living subjects. ${ }^{36-38}$ Recently, it has been reported that Per2 is involved in abnormal cell proliferation, carcinogenesis, and development of malignant tumors, including breast cancer, prostate cancer, colorectal carcinoma, and glioma. ${ }^{39-41}$ With the aim of investigating the role of Per2 in chronic chemotherapy for NSCLC, we performed RTPCR and Western blotting to detect the gene and protein expression of Per2 in NSCLC and tumorous tissues. We studied the relationship between the expression levels of Per2 and timely chemotherapy. We discovered the gene and protein expression of Per2 gradually increased and then decreased. The mRNA and protein expression of Per2 was the highest at 15 HALO. In addition, we performed RT-PCR analysis to investigate the gene expression of Bax and $\mathrm{Bcl}-2$ at specific administration times. Our data revealed that the mRNA expression of Bax was consistent with that of Per2, while the mRNA expression of Bcl-2 was opposite to that of Bax mRNA in vivo. However, in our study, the tumor volume of the PTX group and PTX-NPs group has no significant difference. It is because that PTX-NPs delivery system works for a long time. The PTX-NPs delivery was slower than PTX. Moreover, our results demonstrated that PTX-NPs have a better therapeutic effect than PTX, with reduced organ damage.

Per2 acts as a tumor suppressor in NSCLC carcinogenesis. In our study, we demonstrated that chemotherapy with PTX-NPs had the best therapeutic effect at 15 HALO. Therefore, our work provides evidence that appropriate chemotherapy timing has better therapeutic efficacy which might regulate of Per2 expression. Our research can help to further optimize therapeutic strategies for lung cancer. Moreover, the correlation between chemotherapeutic effects of PTX-NPs and Per2, the definitive relationship will be further established by Per2 gene deficiency in the next research. 
A

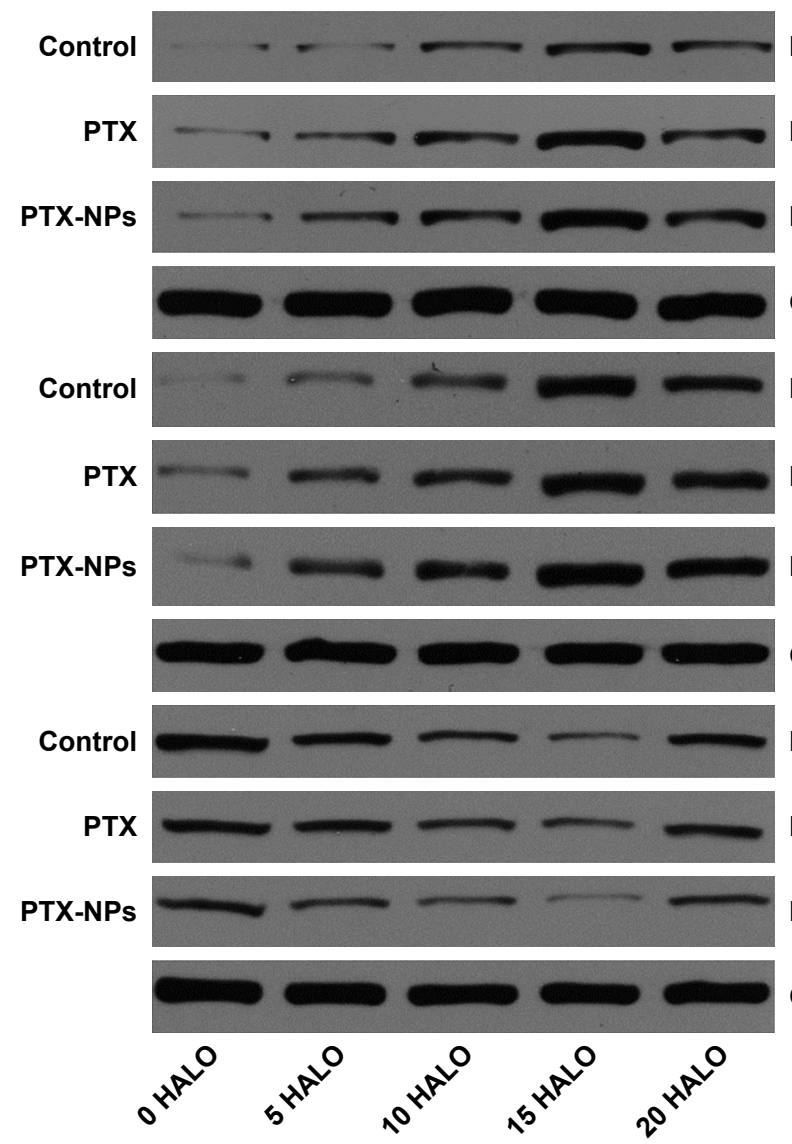

Per2

Per2

Per2

GAPDH

Bax

Bax

Bax

GAPDH

$\mathrm{Bcl}-2$

Bcl-2

Bcl-2

GAPDH
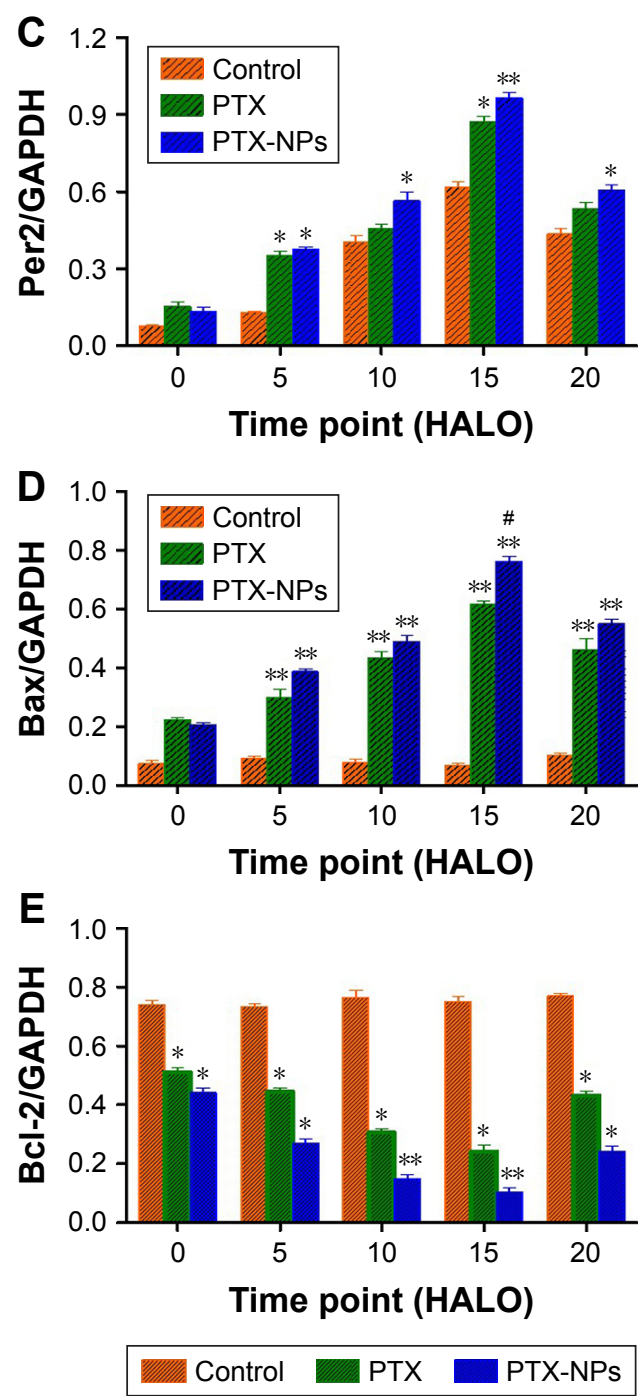

B

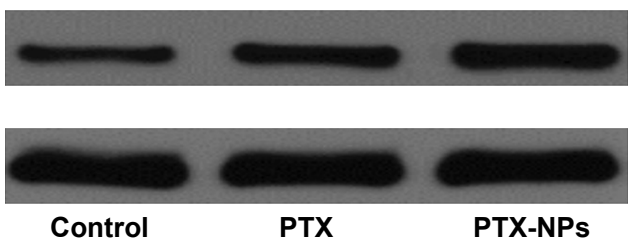

Per2

GAPDH

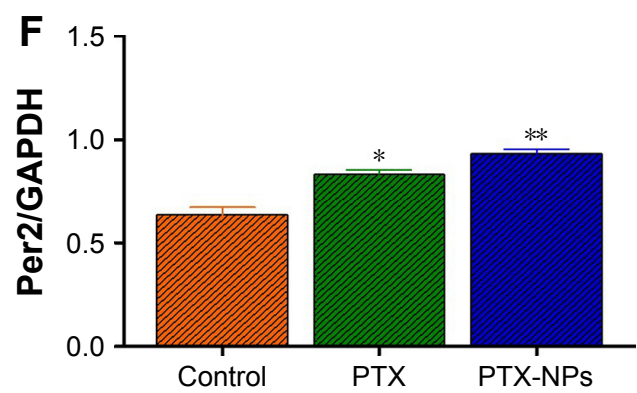

Figure 6 The protein expression of Per2, Bax, and Bcl-2 after PTX-NPs treatment at 0, 5, 10, 15, and 20 HALO.

Notes: (A) Per2, Bax, and Bcl-2 expression in A549-transplanted null mice was determined by Western blotting analysis; (B) The protein expression of Per2 after PTX-NPs treatment at I5 HALO; (C-E) The protein expression level of Per2, Bax, and Bcl-2 were analyzed by Imagej software; (F) the protein expression level of Per2 were analyzed at 15 HALO by Imagej software. GAPDH was used as an internal control. Compared to control, ${ }^{* P}<0.05$, and $* * P<0.01$; compared to PTX, ${ }^{P}<0.05$. Mice treated with saline were used as controls.

Abbreviations: HALO, hours after light onset; PTX, paclitaxel; PTX-NPs, paclitaxel nanoparticles.

\section{Conclusion}

In our study, we investigated Per2-regulated cell death in chronic chemotherapy. PTX-NPs were prepared via a thin film dispersion method. PTX and PTX-NPs might induce apoptosis and block the cell cycle in A549 cells.
PTX and PTX-NPs exhibit tumor growth inhibition rate in A549-transplanted null mice. In addition, PTX and PTXNPs exert the most effective antitumor activity at 15 HALO in vivo and in vitro. Our study demonstrates that PTX and PTX-NPs exhibit anticancer effects by upregulating Per2 

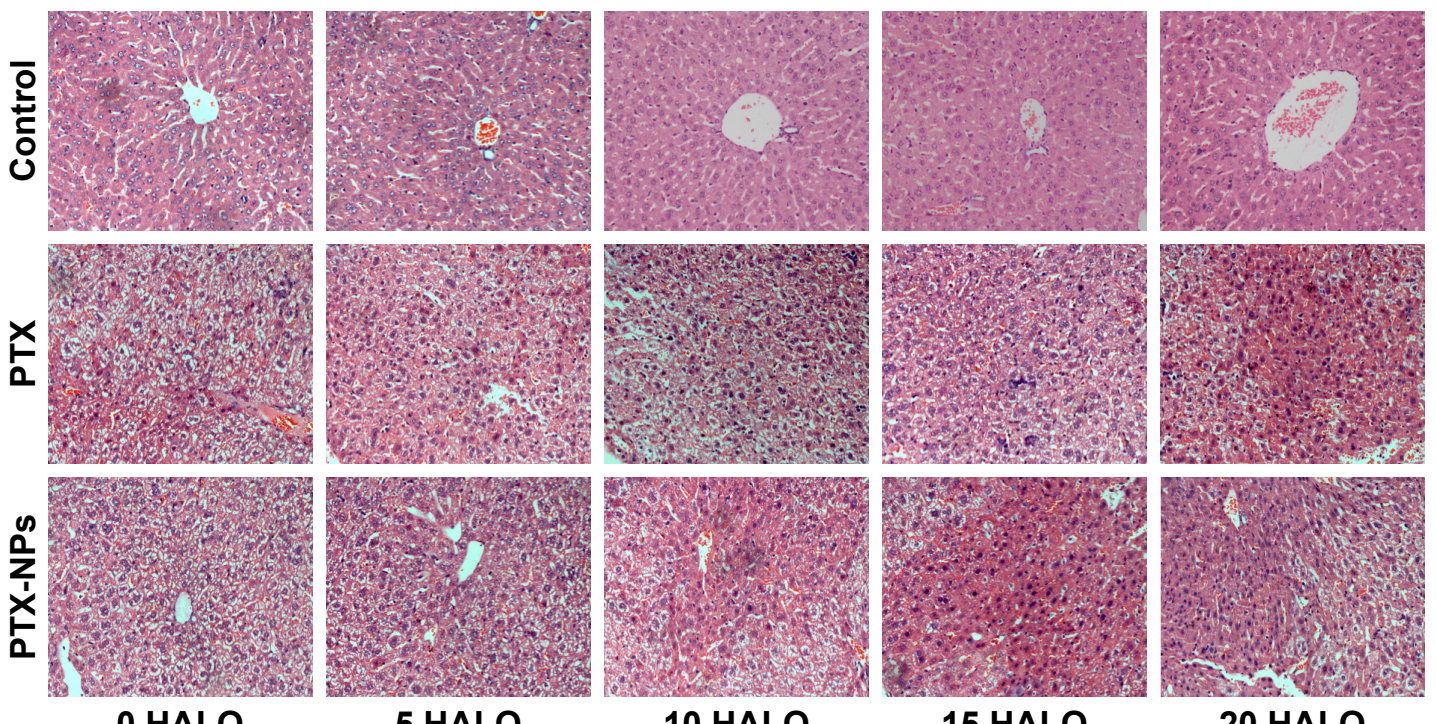

10 HALO

15 HALO

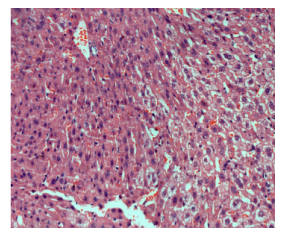

20 HALO

Figure 7 Liver damage after PTX-NPs treatment of A549-transplanted null mice at 0, 5, 10, 15, and 20 HALO (400X).

Abbreviations: HALO, hours after light onset; PTX, paclitaxel; PTX-NPs, paclitaxel nanoparticles.

gene and protein expression and regulating Bax and Bcl-2 expression to induce cell death.

\section{Acknowledgment}

This work was financially supported by the National Natural Science Foundation of China (NSFC81201784).

\section{Disclosure}

The authors report no conflicts of interest in this work.

\section{References}

1. Cheng TY, Cramb SM, Baade PD, Youlden DR, Nwogu C, Reid ME. The International epidemiology of lung cancer: latest trends, disparities, and tumor characteristics. $J$ Thorac Oncol. 2016;11(10):1653-1671.

2. Kawaguchi T, Koh Y, Ando M, et al. Prospective analysis of oncogenic driver mutations and environmental factors: Japan molecular epidemiology for lung cancer study. J Am Soc Clin Oncol. 2016;34(19): 2247-2257.

3. Molina JR, Yang P, Cassivi SD, Schild SE, Adjei AA. Non-small cell lung cancer: epidemiology, risk factors, treatment, and survivorship. Mayo Clin Proc. 2008;83(5):584-594.

4. Didkowska J, Wojciechowska U, Mańczuk M, Łobaszewski J. Lung cancer epidemiology: contemporary and future challenges worldwide. Ann Transl Med. 2016;4(8):150.

5. Hoffman PC, Mauer AM, Vokes EE. Lung cancer. Lancet. 2000; 355(9202):479-485.

6. Sarihan S, Kayisogullari U, Ercan I, Engin K. Randomized phase 2 study of radiotherapy alone versus radiotherapy with paclitaxel in non-small cell lung cancer. $J$ Int Med Res. 2004;32(4):375-383.

7. Sparano JAJ, Wang M, Martino S. Weekly paclitaxel in the adjuvant treatment of breast cancer. J Evid-Based Med. 2009;359(3):310.

8. Fujii T, Tachibana M, Dhar DK, et al. Combination therapy with paclitaxel and thalidomide inhibits angiogenesis and growth of human colon cancer xenograft in mice. Anticancer Res. 2003;23(3B):2405-2411.

9. Wu ZH, Lu MK, Hu LY, Li X, Zh W, Ly HU. Praziquantel synergistically enhances paclitaxel efficacy to inhibit cancer cell growth. PLoS One. 2012;7(12):e51721.
10. de Souza R, Zahedi P, Allen CJ, Piquette-Miller M. Polymeric drug delivery systems for localized cancer chemotherapy. Drug Deliv. 2010;17(6):365-375.

11. Mora-Huertas CE, Fessi H, Elaissari A. Polymer-based nanocapsules for drug delivery. Int J Pharm. 2010;385(1-2):113-142.

12. Yadav AK, Agarwal A, Rai G, et al. Development and characterization of hyaluronic acid decorated PLGA nanoparticles for delivery of 5-fluorouracil. Drug Deliv. 2010;17(8):561-572.

13. Yang B, Ni X, Chen L, et al. Honokiol-loaded polymeric nanoparticles: an active targeting drug delivery system for the treatment of nasopharyngeal carcinoma. Drug Deliv. 2017;24(1):660-669.

14. Xi C, Chao L, Kaijiang L. The research on functional mechanism of timely chemotherapy. Mod Prev Med. 2008;35(2):201-202.

15. Junquan Y, Liping L, Ruijuan Z. Perlimary results of concurrent three-dimenonal conformal radio therapy combined with Chronochemotrapy for advanced non-small cell lung cancer. Mod Prev Med. 2006;33(7):1283-1291.

16. Yufeng L, Jun Y. Compare the categories and extents of toxic and side-effect of time chemothrapy and routine chemothrapy. Front Med. 2011;1(21):138-140.

17. Tahara Y, Otsuka M, Fuse Y, Hirao A, Shibata S. Refeeding after fasting elicits insulin-dependent regulation of $P e r 2$ and $R E V$-ERB $\alpha$ with shifts in the liver clock. J Biol Rhythms. 2011;26(3):230-240.

18. Lévi F, Filipski E, Iurisci I, Li XM, Innominato P. Cross-talks between circadian timing system and cell division cycle determine cancer biology and therapeutics. Cold Spring Harb Symp Quant Biol. 2007;72(1):465-475.

19. Tsimakouridze EV, Alibhai FJ, Martino TA. Therapeutic applications of circadian rhythms for the cardiovascular system. Front Pharmacol. 2015;6:77.

20. Roenneberg T, Daan S, Merrow M. The art of entrainment. $J$ Biol Rhythms. 2003;18(3):183-194.

21. Zhang YH, Zhao GF, Yin KF. The expression and clinical significance of $\beta$-galactose lectin 3 and clock genes Per2 in non-small cell lung cancer. Chinese J Heal Lab Tech. 2014;24(18):2692-2694.

22. Yang X, Wood PA, Oh EY, Du-Quiton J, Ansell CM, Hrushesky WJ. Down regulation of circadian clock gene period 2 accelerates breast cancer growth by altering its daily growth rhythm. Breast Cancer Res Treat. 2009;117(2):423-431.

23. Hu J, Fu S, Peng Q, et al. Paclitaxel-loaded polymeric nanoparticles combined with chronomodulated chemotherapy on lung cancer: in vitro and in vivo evaluation. Int J Pharm. 2017;516(1-2):313-322. 
24. Park CW, Bak Y, Kim MJ, et al. The novel small molecule STK899704 promotes senescence of the human A549 NSCLC cells by inducing DNA damage responses and cell cycle arrest. Front Pharmacol. 2018;9:163.

25. Gadgeel SM. Personalized therapy of non-small cell lung cancer (NSCLC). Adv Exp Med Biol. 2016;890(3):203.

26. Hope WW, Bolton WD, Kalbaugh CA, Blackhurst DW, Stephenson JE, Taylor SM. Lung cancer resection in octogenarians: a reasonable approach for our aging population. Am Surg. 2007;73(1):22.

27. Frings S. New molecular targeted therapeutic drugs clinical results of bevacizumab in non-small cell lung cancer (NSCLC). Haigan. 2006; 46(3):277-281.

28. Abbasi S, Badheeb A. Prognostic factors in advanced non-small-cell lung cancer patients: patient characteristics and type of chemotherapy. Lung Cancer Int. 2011;2011(4):1-4.

29. Huang CY, Ju DT, Chang CF, Muralidhar Reddy P, Velmurugan BK. A review on the effects of current chemotherapy drugs and natural agents in treating non-small cell lung cancer. Biomedicine. 2017;7(4):23.

30. Chen K, Shi W. Autophagy regulates resistance of non-small cell lung cancer cells to paclitaxel. Tumor Biol. 2016;37(8):10539-10544.

31. Coyne PJ, Wan W, Dodson P, Swainey C, Smith TJ. A trial of Scrambler therapy in the treatment of cancer pain syndromes and chronic chemotherapy-induced peripheral neuropathy. J Pain Palliat Care Pharmacother. 2013;27(4):359-364.

32. Wang TH, Wang HS, Soong YK. Paclitaxel-induced cell death: Where the cell cycle and apoptosis come together. Cancer. 2000;88(11): 2619-2628.

33. Chang YF, Li LL, Wu CW, et al. Paclitaxel-induced apoptosis in human gastric carcinoma cell lines. Cancer. 1996;77(1):14-18.
34. Morales-Cano D, Calviño E, Rubio V, et al. Apoptosis induced by paclitaxel via Bcl-2, BAX and caspases 3 and 9 activation in NB4 human leukaemia cells is not modulated by ERK inhibition. Exp Toxicol Pathol. 2013;65(7-8):1101-1108.

35. Salah-Eldin AE, Inoue $\mathrm{S}$, Tsukamoto $\mathrm{S}$, Aoi H, Tsuda M. An association of Bcl-2 phosphorylation and Bax localization with their functions after hyperthermia and paclitaxel treatment. Int J Cancer. 2003; 103(1):53-60

36. Yang X, Wood PA, Ansell CM, et al. The circadian clock gene Per1 suppresses cancer cell proliferation and tumor growth at specific times of day. Chronobiol Int. 2009;26(7):1323-1339.

37. Zhao N, Yang K, Yang G, et al. Aberrant expression of clock gene Period 1 and its correlations with the growth, proliferation and metastasis of buccal squamous cell carcinoma. PLoS One. 2013;8(2):e55894.

38. Xu Y, Toh KL, Jones CR, Shin JY, Fu YH, Ptácek LJ. Modeling of a human circadian mutation yields insights into clock regulation by PER2 Cell. 2007;128(1):59-70.

39. Su X, Chen D, Yang K, et al. The circadian clock gene PER2 plays an important role in tumor suppression through regulating tumor-associated genes in human oral squamous cell carcinoma. Oncol Rep. 2017; 38(1):472-480.

40. Chen ST, Choo KB, Hou MF, Yeh KT, Kuo SJ, Chang JG. Deregulated expression of the PER1, PER2 and PER3 genes in breast cancers. Carcinogen. 2005;26(7):1241-1246.

41. Winter SL, Bosnoyan-Collins L, Pinnaduwage D, Andrulis IL. Expression of the circadian clock genes Per1 and Per2 in sporadic and familial breast tumors. Neoplasia. 2007;9(10):797-800.
International Journal of Nanomedicine

\section{Publish your work in this journal}

The International Journal of Nanomedicine is an international, peerreviewed journal focusing on the application of nanotechnology in diagnostics, therapeutics, and drug delivery systems throughou the biomedical field. This journal is indexed on PubMed Central, MedLine, CAS, SciSearch $\AA$, Current Contents $₫ /$ Clinical Medicine,

\section{Dovepress}

Journal Citation Reports/Science Edition, EMBase, Scopus and the Elsevier Bibliographic databases. The manuscript management system is completely online and includes a very quick and fair peer-review system, which is all easy to use. Visit http://www.dovepress.com/ testimonials.php to read real quotes from published authors. 